\title{
VDAC1 is regulated by BRD4 and contributes to JQ1 resistance in breast cancer
}

\author{
GUOCHAO YANG ${ }^{1 *}$, DIANWEI ZHOU ${ }^{2 *}, \mathrm{JUN} \mathrm{LI}^{3}$, WEI WANG ${ }^{4}$, WEI ZHONG ${ }^{4}$, \\ WEI FAN ${ }^{4}$, MANCHENG YU ${ }^{4}$ and HONGTAO CHENG ${ }^{4}$
}

\author{
${ }^{1}$ Department of General Surgery, Rongjun Hospital, Wuhan, Hubei 430079; ${ }^{2}$ Department of Gastrointestinal Surgery, \\ Xianning Central Hospital, The First Affiliated Hospital of Hubei University of Science and Technology; \\ ${ }^{3}$ Department of Surgery, Clinical Medical College, Hubei University of Science and Technology, Xianning, \\ Hubei 437100; ${ }^{4}$ Department of Breast Surgery, Hubei Cancer Hospital, Wuhan, Hubei 430079, P.R. China
}

Received August 18,2018; Accepted May 29, 2019

\section{DOI: $10.3892 / \mathrm{ol} .2019 .10534$}

\begin{abstract}
Voltage-dependent anion channels (VDACs) are situated in the outer membrane of the mitochondria and serve as gatekeepers that control metabolite and ion exchange between the cytosol and mitochondria. VDAC1 is one of the most studied members of the VDAC protein family and is overexpressed in multiple types of cancer. However, the specific biological function and regulatory mechanism of VDAC1 in breast cancer remains unclear. The present study investigated the biological role of VDAC1 in breast cancer cells using an MTS assay. The association of clinicopathological features with VDAC1 in breast cancer was analyzed by Gene Expression Profiling Interactive Analysis. The regulatory mechanism of VDAC1 was determined by cell transfection, western blot analysis, reverse transcription-quantitative (q)PCR analysis, chromatin immunoprecipitation (ChIP) and ChIP-qPCR analysis. The results of the present study demonstrated that VDAC1 promoted breast cancer proliferation and was associated with a poor prognosis in patients with breast cancer. Additionally, it was observed that the expression of VDAC1 could be decreased by the bromodomain inhibitor (JQ1), and bromodomain-containing protein 4 (BRD4) was indicated to be a regulator of VDAC1. Furthermore, results suggested that VDAC1 may be involved in the resistance of breast cancer to JQ1. Collectively, the present findings uncovered important aspects of the function of VDAC1 in the tumor progression of
\end{abstract}

Correspondence to: Dr Hongtao Cheng, Department of Breast Surgery, Hubei Cancer Hospital, 116 Zhoudaoquan South Road, Wuhan, Hubei 430079, P.R. China

E-mail: 334633124@qq.com

"Contributed equally

Key words: voltage-dependent anion channel 1, bromodomaincontaining protein 4 , bromodomain inhibitors, drug resistance, breast cancer breast cancer, and may provide a basis for potential therapeutic strategies for the treatment of breast cancer.

\section{Introduction}

Breast cancer is the most common cancer and the leading cause of mortality among women worldwide (1). Cancer cells are characterized by their state of higher proliferation compared with normal cells (2). This requires reprogramming of cell metabolism and is thus associated with the mitochondria, the site of ATP generation (3). Therefore, mitochondrial bioenergetics, biosynthesis and signaling are involved in tumorigenesis (4).

Metabolites and ions are exchanged between the cytosol and mitochondria via the mitochondrial outer membrane, and this process is controlled by mitochondrial porins, also known as voltage-dependent anion channels (VDACs) (5). VDAC1 is one of the most studied members of the VDAC protein family and is located in the outer mitochondrial membrane (5). VDAC1 functions as a mitochondrial gatekeeper and controls the exchange of metabolites, fatty acid ions, calcium ions $\left(\mathrm{Ca}^{2+}\right)$, reactive oxygen species (ROS) and cholesterol between the cytosol and mitochondria (6). Additionally, VDAC1 interacts with numerous proteins of the cytosol, endoplasmic reticulum and mitochondria to regulate metabolic processes, cell proliferation and apoptosis (7).

It has been documented that VDAC1 is overexpressed in multiple types of cancer, including liver cancer (8). non-small cell lung cancer (9). and cervical cancer (10). and that it promotes tumor growth and apoptosis $(7,8)$. However, the specific biological function and regulatory mechanism of VDAC1 in breast cancer has remained unclear. In the present study, the effect of VDAC1 on the growth of breast cancer and its association with the clinicopathological features of patients with breast cancer were assessed. VDAC1 may be an ideal pharmacological target for cancer therapy (11). Numerous antitumor small molecule inhibitors have been designed to interact with VDAC1 and block its function (12). Bromodomain inhibitors exhibit antitumor activity via epigenetic upregulation and downregulation of gene expression in breast cancer (13). JQ1 is one of the most commonly studied bromodomain inhibitors. 
JQ1 can block the function of bromodomain-containing proteins (BRDs), including BRD4, BRD2 and BRD3 (14). In particular, BRD4 is the major bromodomain containing protein established to promote tumorigenesis (15). Therefore, the effects of a bromodomain inhibitor (JQ1) and the role of BRD4 on the regulation of VDAC1 were investigated in breast cancer cell lines in vitro. Bromodomain inhibitors have been shown to inhibit breast cancer proliferation in vitro and in vivo (16). However, acquired drug resistance can reduce the antitumor effect of these small molecule inhibitors. It has been reported that several genes, including c-Myc and speckle-type POZ domain protein, may be crucial for the resistance of cancer cells to bromodomain inhibitors $(14,17)$. The role of VDAC1 in the resistance of breast cancer to JQ1 was also examined. Taken together, the current data may contribute to an improved understanding of the role of VDAC1 in breast cancer and provide a basis for potential therapeutics targeted at breast cancer.

\section{Materials and methods}

Cell culture. The breast cancer MCF-7 and T47D cell lines were purchased from the Chinese Academy of Sciences Cell Bank. The 293T cells were purchased from Procell Life Science and Technology Co., Ltd. MCF-7, T47D and 293T cells were cultured in Dulbecco's modified Eagle's medium (Invitrogen; Thermo Fisher Scientific, Inc.) with $10 \%$ fetal bovine serum (HyClone; GE Healthcare Life Sciences). The cell lines were routinely maintained at $37^{\circ} \mathrm{C}$ and in an atmosphere of $5 \%$ $\mathrm{CO}_{2}$. JQ1 was purchased from Selleck Chemicals. JQ1 was dissolved in DMSO, and an equal amount of DMSO was used as the control.

Cell transfection. The breast cancer cells (MCF-7 and T47D; $1 \times 10^{6}$ cells) were transfected with Flag-BRD4 plasmids $(2 \mu \mathrm{g}$; cat. no. 90331; Addgene, Inc.) or empty vector (EV; pcDNA3.1; $2 \mu \mathrm{g}$ ) using Lipofectamine ${ }^{\circledR} 2000$ (Invitrogen; Thermo Fisher Scientific, Inc.) according to the manufacturer's protocol. At $24 \mathrm{~h}$ post-transfection, cells were collected for further analysis. The transfection efficiency was determined by western blot analysis.

Western blot analysis. Whole cell lysates were prepared by resuspending cells in whole cell lysis buffer containing $0.5 \%$ NP-40 and $1 \%$ SDS, with $1 \%$ protease and phosphatase inhibitors. Protein concentration was determined using a bicinchoninic acid assay. Total protein from each cell group (80 $\mu \mathrm{g} /$ lane) was loaded and separated on $10 \%$ SDS-PAGE gels and transferred onto PVDF membranes (Pierce; Thermo Fisher Scientific, Inc.). The membranes were blocked with 5\% skimmed milk in $1 \mathrm{X}$ TBST $[10 \mathrm{mmol} / \mathrm{l}$ Tris- $\mathrm{HCl}(\mathrm{pH} 7.4)$, $150 \mathrm{mmol} / 1 \mathrm{NaCl}$ and $0.1 \%$ Tween 20] and incubated with primary antibodies overnight at $4^{\circ} \mathrm{C}$. The membranes were then washed with $1 \mathrm{X}$ TBST and incubated with a secondary antibody for $1 \mathrm{~h}$ at room temperature. Finally, the membranes were treated with electrochemiluminescence detection reagents (SuperSignal West Pico Stable Peroxide solution; Thermo Fisher Scientific, Inc.) and exposed to X-ray film. ImageJ software (ImageJ bundled with Java version 1.8.0_112; National Institutes of Health) was used for semi-quantification of protein levels. The primary antibodies used were against VDAC1 (cat. no. ab14734; Abcam; dilution, 1:1,000), Flag (cat. no. A5712; Bimake; dilution, 1:2,000) and BRD4 (cat. no. 13440; Cell Signaling Technology, Inc.; dilution, 1:1,000). Anti- $\beta$-tubulin antibody (cat. no. 2148; Cell Signaling Technology, Inc.; dilution, 1:2,000) was used as a loading control. The secondary antibodies were peroxidase IgG fraction monoclonal mouse anti-rabbit IgG (cat. no. 211-032-171; Jackson ImmunoResearch Laboratories, Inc.; dilution, 1:5,000) and peroxidase AffiniPure goat anti-mouse IgG (cat. no. 115-035-174; Jackson ImmunoResearch Laboratories, Inc.; dilution, 1:5,000).

Reverse transcription-quantitative polymerase chain reaction $(R T-q P C R)$. MCF-7 and T47D cells transfected with shControl, shVDAC1, shBRD4, EV (pcDNA3.1) or Flag-BRD4 constructs, and treated with DMSO or JQ1 were subjected to RT-qPCR analysis. Total RNA was extracted from these cells using TRIzol ${ }^{\circledR}$ reagent (Thermo Fisher Scientific, Inc.). The cDNA was synthesized using Superscript II reverse transcriptase (Thermo Fisher Scientific, Inc.), dNTP mix (cat. no. 18427088; Thermo Fisher Scientific, Inc.), random hexamers (cat. no. N8080127; Thermo Fisher Scientific, Inc.) and $5 \mathrm{X}$ first-strand buffer (Thermo Fisher Scientific, Inc.). The following temperature protocol was used: First step (denaturation at $70^{\circ} \mathrm{C}$ for $10 \mathrm{~min}$, followed by $4^{\circ} \mathrm{C}$ for $10 \mathrm{~min}$ ); second step $\left(42^{\circ} \mathrm{C}\right.$ for $50 \mathrm{~min}$, denaturing at $85^{\circ} \mathrm{C}$ for $10 \mathrm{sec}$, $4^{\circ} \mathrm{C}$ for $\left.10 \mathrm{~min}\right)$. qPCR was performed using IQ SYRB Green Supermix and an iCycleriQTX detection system (Bio-Rad Laboratories, Inc.). The following thermocycling conditions were used: Initial denaturation at $95^{\circ} \mathrm{C}$ for $60 \mathrm{sec}$, denaturing at $95^{\circ} \mathrm{C}$ for $20 \mathrm{sec}$, annealing at $58^{\circ} \mathrm{C}$ for $30 \mathrm{sec}$ and extension at $72^{\circ} \mathrm{C}$ for $30 \mathrm{sec}$, for 43 cycles, followed by melting curve at $65-95^{\circ} \mathrm{C}$ with increments of $0.5^{\circ} \mathrm{C}$ for $5 \mathrm{sec}$. All signals were normalized to GAPDH and the $2^{-\Delta \Delta \mathrm{Cq}}$ method was used to quantify the fold-change (18). The primers used were as follows: VDAC1 forward, 5'-GGTGCTCTGGTGCTAGGT TA-3' and reverse, 5'-CAGCGGTCTCCAACTTCTTG-3'; BRD4 forward, 5'-ACAACCCTCCTGACCATGAG-3' and reverse, 5'-AACTGTCACTGTCCGAGGAG-3'; and GAPDH forward, 5'-ACCCACTCCTCCACCTTTGAC-3' and reverse, 5'-TGTTGCTGTAGCCAAATTCGTT-3'.

RNA interference. Lentivirus vector (Tet-pLKO-puroScrambled; cat. no. 47541; Addgene, Inc.) and lentivirus-based gene-specific short hairpin (sh)RNAs were purchased from Sigma-Aldrich [cat. nos. SHCLNG-NM_003374 for VDAC1 (shVDAC1\#1, TRCN0000278508; shVDAC1\#2, TRCN0000029125) and SHCLNG-NM_058243 for BRD4 (shBRD4\#1, TRCN0000088478; shBRD4\#2, TRCN0000088481)]. Transfections were performed using Lipofectamine 2000. A total of $2 \mu \mathrm{g}$ gene-specific shRNA or shNT (control) was transfected into $293 \mathrm{~T}$ cells $\left(5 \times 10^{5}\right.$ cells; cat. no. C004; Wuhan Fine Biotech Co., Ltd.). After 48 h, the culture medium of the $293 \mathrm{~T}$ cells was collected $(10,000$ viral particles $/ \mu \mathrm{l}$ ) and $10 \mu \mathrm{l}$ viral medium was applied to 100,000 MCF-7 and T47D breast cancer cells. Breast cancer cells were cultured in $5 \% \mathrm{CO}_{2}$. at $37^{\circ} \mathrm{C}$ for $48 \mathrm{~h}$, followed by puromycin $(0.75 \mu \mathrm{g} / \mathrm{ml}$; Sigma-Aldrich; Merck KGaA) selection for another $24 \mathrm{~h}$. Cells were collected at $72 \mathrm{~h}$ 
post-transfection $(48 \mathrm{~h}$ of infection and $24 \mathrm{~h}$ of puromycin selection). The knockdown efficiency was confirmed through western blotting using the aforementioned method. The shRNA sequences were as follows: shNT, 5'-CCTAAGGTT AAGTCGCCCTCGCTCGAGCGAGGGCGACTTAACCTT AGG-3' (Tet-pLKO-puro-Scrambled); shVDAC1\#1, 5'-CCG GCAAGTACAGATGGACTGAGTACTCGAGTACTCAGT CCATCTGTACTTGTTTTTG-3'; shVDAC1\#2, 5'-CCGGCG ATTCATCCTTCTCACCTAACTCGAGTTAGGTGAGAA GGATGAATCGTTTTT-3'; shBRD4\#1, 5'-CCGGGACTC CATCAAGTTATGGAATCTCGAGATTCCATAACTTGA TGGAGTCTTTTTG-3'; and shBRD4\#2, 5'-CCGGGATGT GTTTGAAATGCGCTTTCTCGAGAAAGCGCATTTCAA ACACATCTTTTTG-3'.

Cell proliferation assay. Cell proliferation was monitored using an MTS assay (Promega Corporation) according to the manufacturer's protocols. The MCF-7 and T47D cells were infected with shNT (control) or VDAC1-specific shRNA, as previously described. At $24 \mathrm{~h}$ post-transfection, the cells were placed on 96-well plates at a density of 3,000 cells/well. Cells were treated with different concentrations $(0,0.625,1.25,2.5,5,10$, 20, 40, 80 and $160 \mu \mathrm{M}$ ) of JQ1 for $24 \mathrm{~h}$. Next, $20 \mu$ l CellTiter $^{\circledR}$ 96R AQueous One Solution Reagent (Promega Corporation) was added to each well. After $50 \mathrm{~min}$ of incubation at $37^{\circ} \mathrm{C}$ in a cell culture incubator, proliferation was measured using a microplate reader at a wavelength of $490 \mathrm{~nm}$.

Survival analysis and correlation analysis using the gene expression profiling interactive analysis (GEPIA) web tool. The online database GEPIA (http://gepia.cancer-pku. $\mathrm{cn} /$ ) (9) was used to analyze the RNA sequencing expression data related to the present project on The Cancer Genome Atlas (TCGA; https://portal.gdc.cancer.gov/) and the Genotype-Tissue Expression (GTEx; V7; http://www. gtexportal.org/home/) projects. GEPIA performed VDAC1 expression analysis based on gene expression levels. For GEPIA analysis, the gene name (VDAC1) was entered on the website, and 'Expression DIY', 'Boxplot' and 'breast cancer' were selected for analysis. VDAC1 expression was compared between breast cancer $(n=1,085)$ and normal breast $(n=291)$ tissues. Tboxplot analysis used $\log 2$ (transcripts per million +1 ) for the log-scale, which was analyzed by the GEPIA web tool. GEPIA performs survival analyses based on gene expression levels and uses a log-rank test for hypothesis evaluation. GEPIA performs a pairwise gene correlation analysis for any given sets of TCGA and/or GTEx expression data using Pearson correlation statistics.

Chromatin immunoprecipitation (ChIP) and ChIP-qPCR. ChIP was performed following the manufacturer's protocol for the Chromatin Extraction kit (cat. no. ab117152; Abcam) and ChIP kit magnetic-one step (cat. no. ab156907, Abcam). The aforementioned anti-BRD4 antibody (dilution, 1:50) was used for the ChIP assay. The purified DNA was analyzed by qPCR using a PCR kit (TB Green ${ }^{\circledR}$ Fast qPCR mix; cat. no. RR430A; Takara Bio, Inc.) according to the manufacturer's protocols. TB Green ${ }^{\circledR}$ was used as the fluorophore. The thermocycling conditions were as follows: Initial denaturation at $95^{\circ} \mathrm{C}$ for $60 \mathrm{sec}$, denaturing at $95^{\circ} \mathrm{C}$ for $20 \mathrm{sec}$, annealing at $58^{\circ} \mathrm{C}$ for
$30 \mathrm{sec}$ and extension at $72^{\circ} \mathrm{C}$ for $30 \mathrm{sec}$, for 43 cycles, followed by melting curve at $65-95^{\circ} \mathrm{C}$ with increments of $0.5^{\circ} \mathrm{C}$ for $5 \mathrm{sec}$. The following primers were used: VDAC1 ChIP primer, forward 5'-TGGGTTCCTGCATCTCTCAG-3' and reverse, 5'-CTTAGGAAGGCTAGGAGGGC-3'. UCSC Genome Browser (http://genome.ucsc.edu/) data (human GRCh37/hg19) was analyzed and processed data were downloaded from the NCBI Gene Expression Omnibus (http://www.ncbi.nlm.nih. gov/geo/) database with the accession number GSE88872 (19).

Statistical analysis. Microsoft Excel software (Microsoft Excel 2013 version 15.19.1; Microsoft Corporation) was used for to perform statistical analysis. Statistical analysis was performed by two-sided paired Student t-tests for comparisons between 2 groups, one-way analysis of variance (ANOVA) with Tukey's multiple comparisons post-hoc test for comparisons of datasets with 1 variable and two-way ANOVA with Bonferroni's post hoc test for comparisons of datasets with $>1$ variable. $\mathrm{P}<0.05$ was considered to indicate a statistically significant difference. Data are presented as the mean \pm standard deviation.

\section{Results}

VDACl promotes cell proliferation and is associated with poor prognosis in breast cancer. As VDAC1 is overexpressed in a number of types of cancer (20-22) and is crucial for the control of proliferation in cancer cells (20), the present study aimed to examine the biological role of VDAC1 in breast cancer. VDAC1 expression was knocked down using two lentivirus-based shRNAs in MCF-7 and T47D cells. Western blotting and RT-qPCR analyses indicated that the protein and mRNA levels of VDAC1 were decreased in the shVDAC1 groups compared with those in the control groups in each cell line (Fig. 1A and B). Additionally, it was observed that knockdown of VDAC1 inhibited proliferation in the two cancer cell lines (Fig. 1C), which was in accordance with the reported effect of VDAC1 in other types of cancer, including prostate (23), lung (22) and colon (21) cancer. To investigate the association of clinicopathological features with VDAC1 in breast cancer, VDAC1 mRNA level in breast cancer and normal breast tissues was assessed using the GEPIA web tool (24). The RT-qPCR data demonstrated that the expression of VDAC1 in breast invasive carcinoma was higher than that in normal breast tissues (Fig. 1D). Next, overall survival and disease-free survival rates associated with breast cancer were analyzed using GEPIA, and it was identified that a high level of VDAC1 expression was associated with poor overall survival rate in breast cancer (Fig. 1E and F). Thus, these findings indicated that VDAC1 is overexpressed in breast cancer and associated with a poor prognosis in breast cancer patients. Additionally, knockdown of VDAC1 promoted breast cancer cell proliferation.

JQ1 decreases VDACl expression in a dose-and timedependent manner. In the current study, breast cancer cells were treated with the bromodomain inhibitor JQ1 (0.5 and $1 \mu \mathrm{M})$ for $24 \mathrm{~h}$, and it was observed that JQ1 treatment led to a decrease in the protein and mRNA levels of VDAC1 in MCF-7 and T47D cells (Fig. 2A and B). It was also observed that JQ1 $(0.5 \mu \mathrm{M})$ could decrease VDAC1 expression in a 

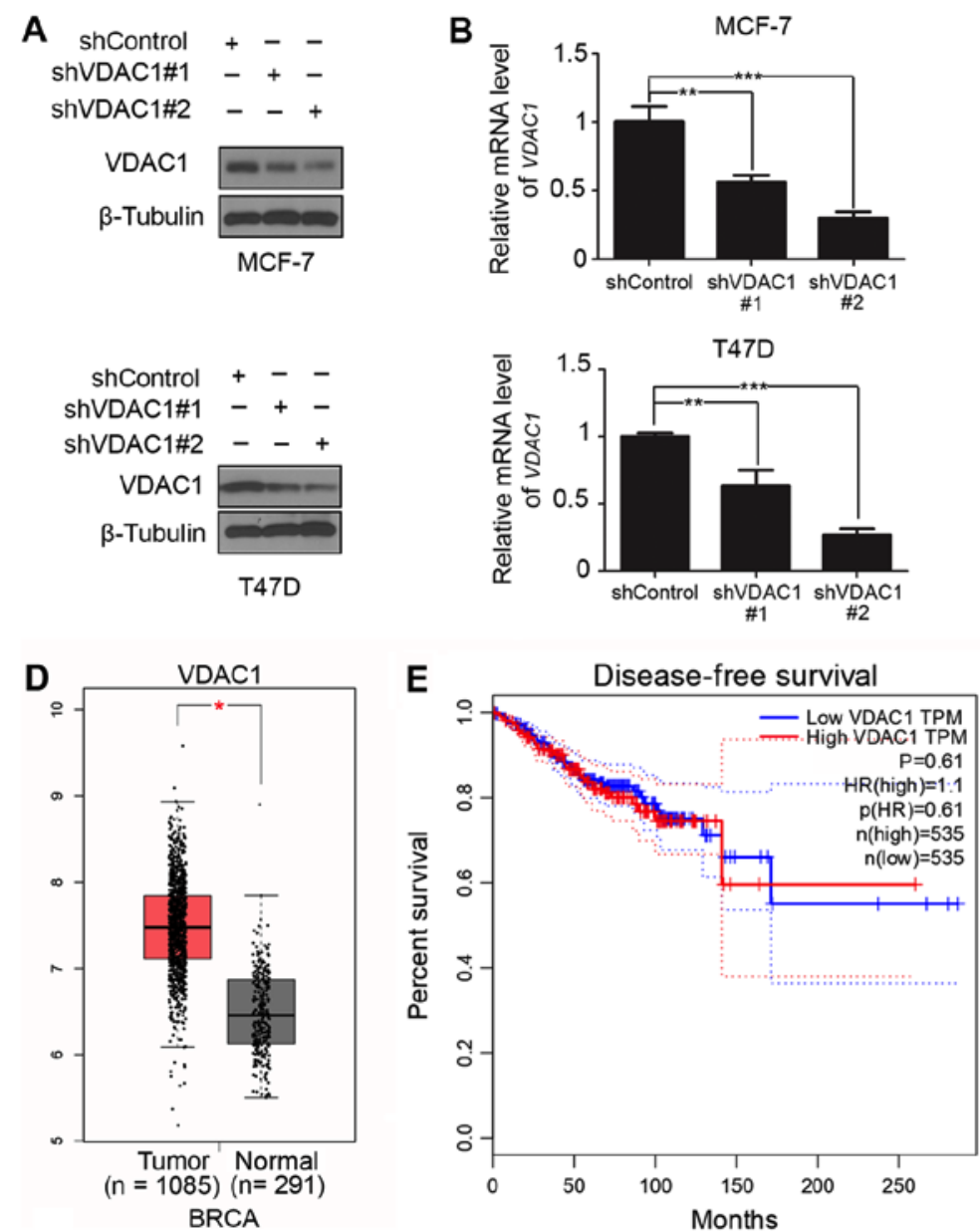
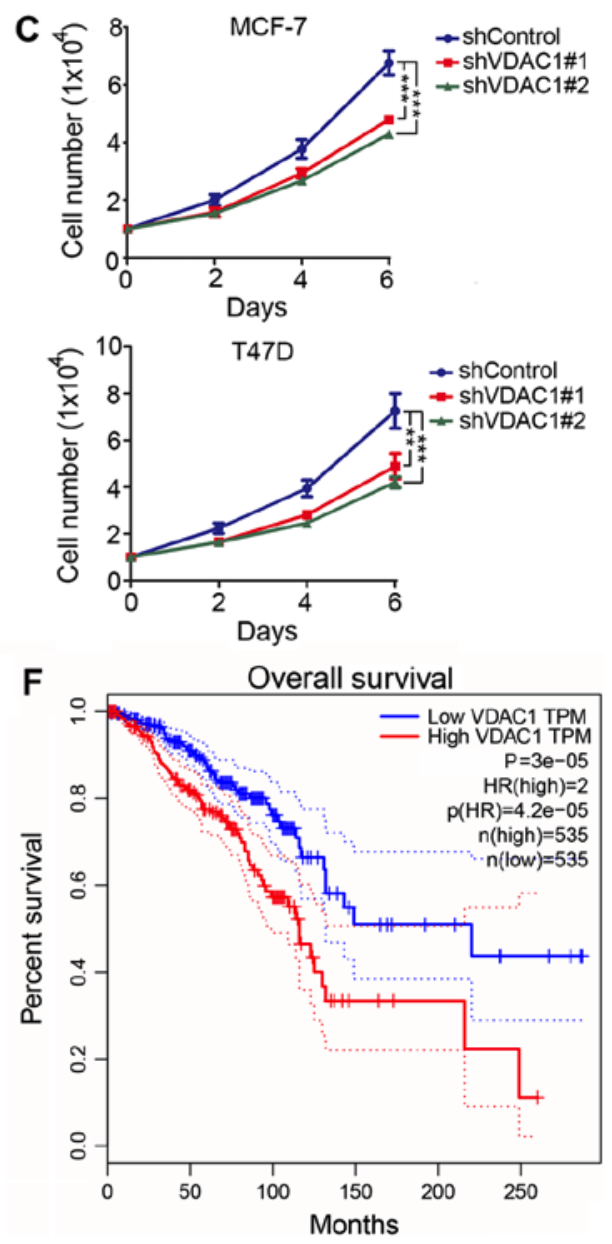

Figure 1. VDAC1 promotes cell proliferation and is associated with a poor prognosis in breast cancer. (A) Western blot analysis, (B) RT-qPCR and (C) cell proliferation assay of human breast cancer cell lines, MCF-7 and T47D, infected by lentivirus encoding shControl, shVDAC1\#1 or shVDAC1\#2 (n=3); ${ }^{*} \mathrm{P}<0.01$, ${ }^{* *} \mathrm{P}<0.01$ and ${ }^{* * *} \mathrm{P}<0.001$. (D) GEPIA database analysis of VDAC1 expression in breast cancer tissue compared with normal tissue. (E) Disease-free survival rate and $(\mathrm{F})$ overall survival rate of breast cancer patients with low or high VDAC1 expression analyzed using log-rank test in GEPIA, and the Mantel-Cox test. The median expression of VDAC1 was selected as the threshold to split the high-expression and low-expression cohorts. VDAC1, voltage-dependent anion-selective channel 1; sh, small hairpin RNA; GEPIA, Gene Expression Profiling Interactive Analysis; TPM, transcripts per million; HR(high), hazard ratio of samples with expression level higher than cut-off threshold; $\mathrm{p}(\mathrm{HR}), \mathrm{P}$-values of hazard ratio; $\mathrm{n}(\mathrm{high})$, number of samples in high expression group; $\mathrm{n}(\mathrm{low})$, number of samples in high expression group.

A
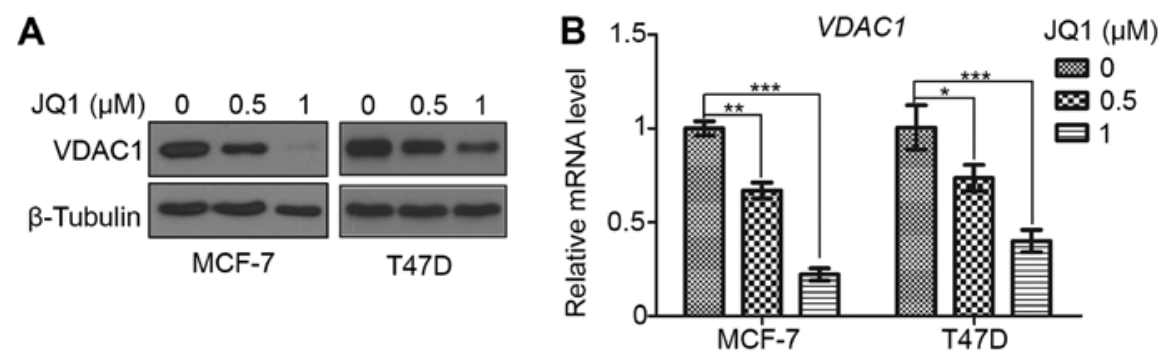

C

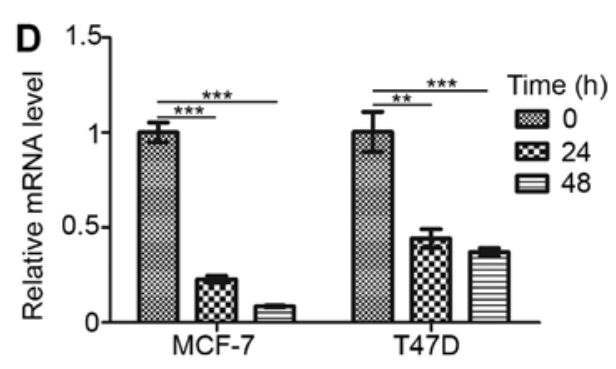

Figure 2. JQ1 decreases VDAC1 expression in a dose- and time-dependent manner. (A) Western blot and (B) RT-qPCR analysis of VDAC1 expression in MCF-7 and T47D cells treated with JQ1 $(0.5$ and $1 \mu \mathrm{M})$ or untreated $(0 \mu \mathrm{M}$; equal amount of DMSO added) for $24 \mathrm{~h}(\mathrm{n}=3)$. (C) Western blot and (D) RT-qPCR analysis of VDAC1 expression in MCF-7 and T47D cells treated with JQ1 $(0.5 \mu \mathrm{M})$ for 24 and $48 \mathrm{~h}$ or untreated $(0 \mu \mathrm{M})$ for $48 \mathrm{~h}(\mathrm{n}=3)$. ${ }^{*} \mathrm{P}<0.05$, ${ }^{* * *} \mathrm{P}<0.01$ and ${ }^{* * * *} \mathrm{P}<0.001$. VDAC1, voltage-dependent anion-selective channel 1; RT-qPCR, reverse transcription-quantitative polymerase chain reaction. 

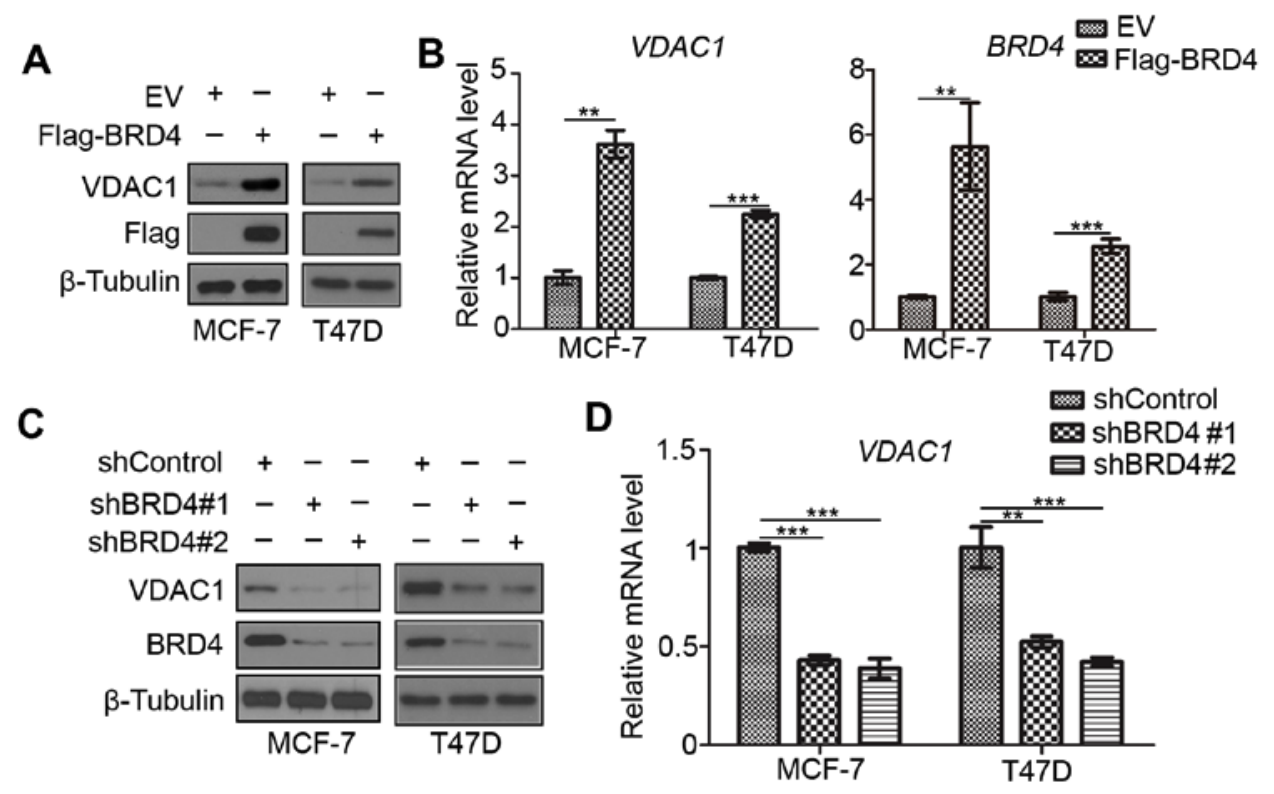

E

$\mathbf{F}$
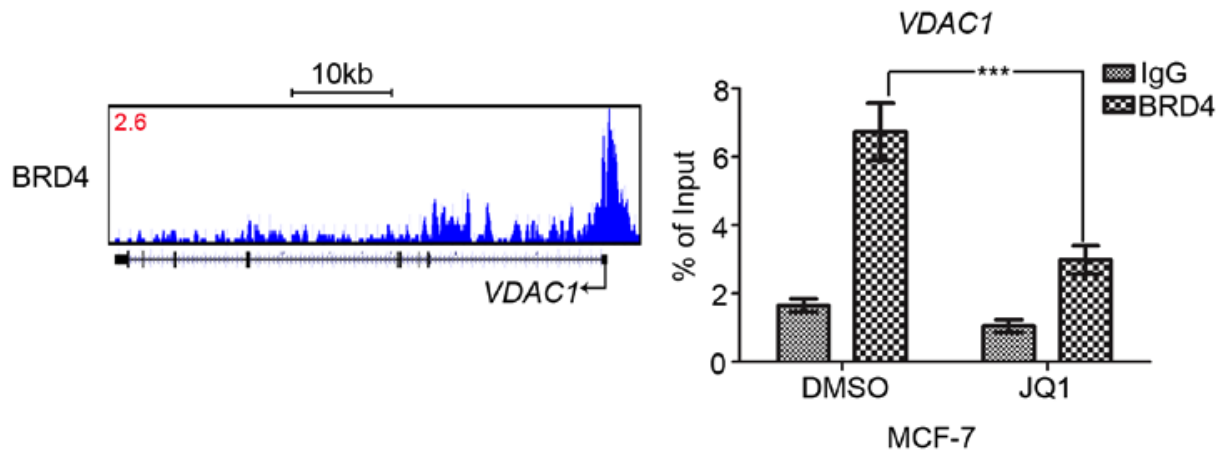

Figure 3. BRD4 increases VDAC1 expression in breast cancer cells. (A) Western blot analysis of VDAC1 and Flag protein expression, and (B) RT-qPCR analysis of VDAC1 and BRD4 mRNA expression in MCF-7 and T47D cells transfected with Flag-BRD4 or control [EV (pcDNA3.1)] (n=3). (C) Western blot analysis of VDAC1 and BRD4 protein expression, and (D) RT-qPCR analysis of VDAC1 mRNA expression in MCF-7 and T47D cells transfected with shControl, shBRD4\#1 or shBRD4\#2 ( $\mathrm{n}=3) .{ }^{* *} \mathrm{P}<0.01$ and ${ }^{* * *} \mathrm{P}<0.001$. (E) UCSC Genome Browser data showing BRD4 ChIP-seq signal profiles in the VDAC1 gene locus. (F) ChIP-qPCR analysis of BRD4 in MCF-7 cells treated with $0.5 \mu \mathrm{M}$ JQ1 or DMSO for $24 \mathrm{~h}$, indicating binding at the VDAC1 promotor (n=3); analysis performed using two-way ANOVA with Bonferroni's post hoc test. ${ }^{* * *} \mathrm{P}<0.001$. BRD4, bromodomain-containing protein 4; EV, empty vector; VDAC1, voltage-dependent anion-selective channel 1; RT-qPCR, reverse transcription-quantitative polymerase chain reaction; sh, small hairpin RNA; ChIP, chromatin immunoprecipitation; DMSO, dimethyl sulfoxide; IgG, immunoglobulin G.

time-dependent manner, with further reductions in protein and mRNA observed at $48 \mathrm{~h}$ compared to $24 \mathrm{~h}$ (Fig. 2C and D). These results indicate that JQ1 can decrease the expression of VDAC1 in breast cancer cells in a dose- and time-dependent manner.

BRD4 increases VDAC1 expression in breast cancer cells. JQ1 inhibits the bromodomain-containing protein BRD4 (14). As VDAC1 expression was decreased by JQ1 treatment in breast cancer cells, the role of BRD4 in the regulation of VDAC1 expression of in breast cancer cells was investigated. Firstly, it was identified that overexpression of BRD4 increased the protein and mRNA levels of VDAC1 in MCF-7 and T47D cells (Fig. 3A and B). Subsequently, knockdown of BRD4 by two distinct shRNAs decreased VDAC1 expression at the protein and mRNA levels in MCF-7 and T47D cells (Fig. 3C and D). Additionally, on analysis of BRD4 ChIP-seq data (19), it was identified that BRD4 bound to the promoter region of VDAC1 (Fig. 3E). This result was further confirmed by ChIP-qPCR in MCF-7 cells (Fig. 3F), which demonstrated that BRD4 could bind to the promoter of VDAC1 and treatment with JQ1 attenuated the binding of BRD4 compared with DMSO treatment. Taken together, these data suggest that BRD4 increases VDAC1 expression in breast cancer cells.

VDAC1 contributes to JQ1 resistance in breast cancer cells. To establish a mechanism underlying the acquired drug resistance of breast cancer cells to bromodomain inhibitors, VDAC1 was knocked down in MCF-7 and T47D cells along with JQ1 $(0.5 \mu \mathrm{M})$ treatment (Fig. 4A). JQ1 decreased the growth of MCF-7 (Fig. 4A) and T47D (Fig. 4B) cells when compared with shControl + DMSO. This effect was enhanced by VDAC1 knockdown at 6 days following transfection and the start of JQ1 treatment. Correspondingly, the $\mathrm{IC}_{50}$ values of JQ1 in MCF-7 (Fig. 4C) and T47D (Fig. 4D) cells with or without knockdown of VDAC1 were determined, and it was observed that knockdown of VDAC1 decreased the $\mathrm{IC}_{50}$ value 
A

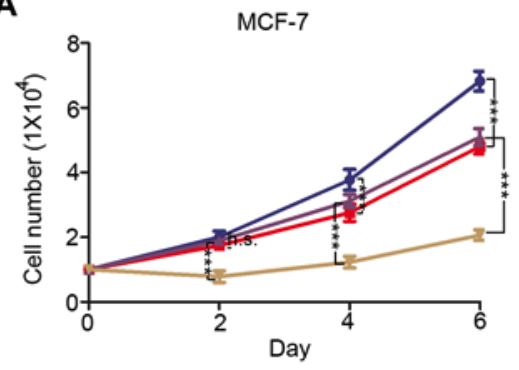

C

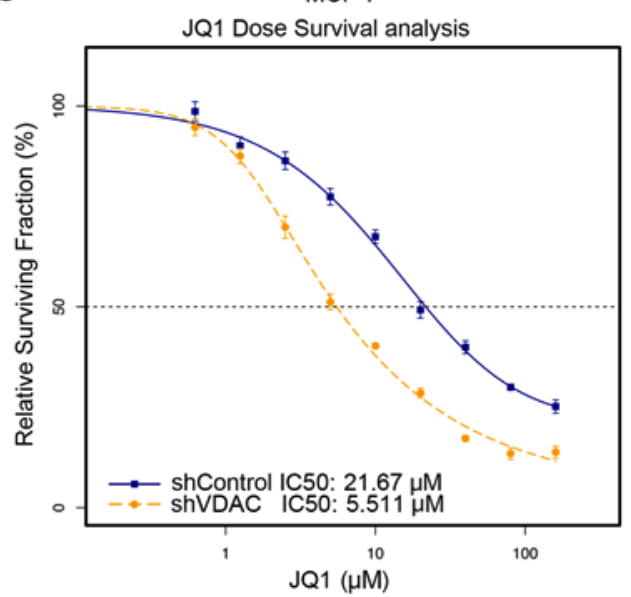

B

$\rightarrow$ shControl + DMSO

- -shVDAC1 + DMSO

\pm shControl + JQ1

$\rightarrow$ ShVDAC1 + JQ1

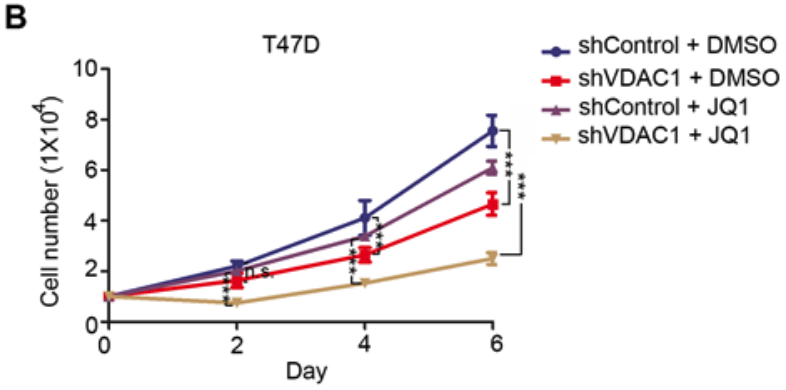

D

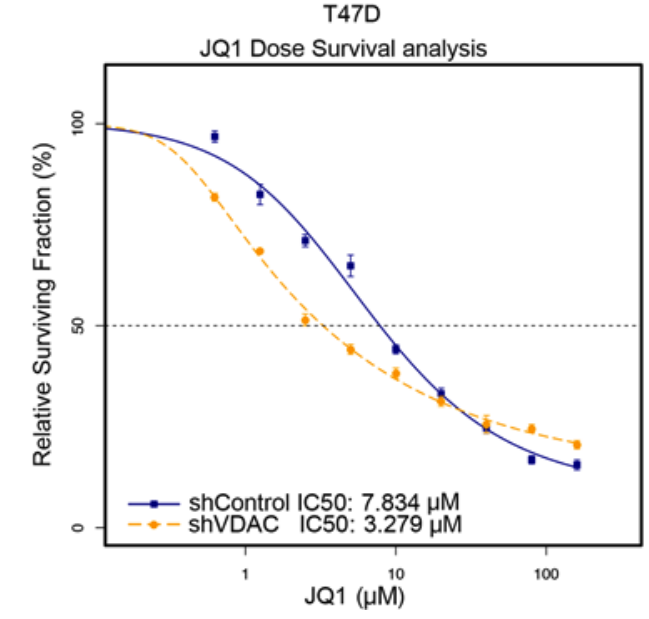

Figure 4. VDAC1 contributes to JQ1 resistance in breast cancer cells. Cell proliferation in (A) MCF-7 cells and (B) T47D cells infected with lentivirus encoding shControl or shVDAC1 for $48 \mathrm{~h}$ followed by treatment with $0.5 \mu \mathrm{M}$ JQ1 or DMSO (n=3) for 0-6 days. At different time points (0, 2,4 or 6 days), cells were harvested for cell number counting; analysis performed using 2 -way ANOVA with Bonferroni post hoc test. ${ }^{* * *} \mathrm{P}<0.001$. Dose-response curves of (C) MCF-7 cells and (D) T47D cells infected with lentivirus encoding shControl or shVDAC1 for $48 \mathrm{~h}$ post-infection followed by treatment with different doses of JQ1 $(0,0.625,1.25,2.5,5,10,20,40,80$ or $160 \mu \mathrm{M})$ for $24 \mathrm{~h}(\mathrm{n}=6)$. VDAC1, voltage-dependent anion-selective channel 1; sh, small hairpin RNA; n.s., not significant.

of JQ1 in MCF-7 and T47D cells. Therefore, these data suggest that VDAC1 may be an important factor in mediating JQ1 resistance in breast cancer cells.

\section{Discussion}

VDAC1 serves as a gatekeeper of the outer mitochondrial membrane by controlling the transfer of metabolites and ions between the cytosol and mitochondria (11). Additionally, VDAC1 also acts as a hub that interacts with numerous proteins, and thereby serves a key role in regulating cell functions, including cell metabolism, $\mathrm{Ca}^{2+}$ homeostasis and apoptosis $(25,26)$. Cancer is a metabolic disease, and mitochondrial dysfunction is a common feature of cancer (27). A previous study reported that the expression of VDAC1 in tumor tissue, including liver cancer (8), non-small cell lung cancer (9) and cervical cancer (10), was higher compared with that in corresponding normal tissue, and that the protein controlled the fate of cancer cells (20). The results of the present study demonstrated that knockdown of VDAC1 inhibited the proliferation of breast cancer cells. Furthermore, the data revealed that expression of VDAC1 was increased in tumor tissue from patients with breast cancer and associated with a poor prognosis. These data indicate that the effect of VDAC1 on breast cancer cells is similar to that reported for other types of cancer.
VDAC1 has been identified as being critical for cancer cell survival $(11,22,28)$. Investigating the specific regulatory mechanism of VDAC1 in cells will not only improve the understanding of the tumorigenic process of cancer cells, but may also provide novel therapeutic strategies for the treatment of breast cancer. A number of anticancer drugs and other cytotoxic agents, including cisplatin (29) and ROS (30), may disrupt $\mathrm{Ca}^{2+}$ homeostasis and increase the expression of VDAC1 in cancer cells (28). VDAC1 can form oligomers that are capable of triggering cytochrome $c$ release and inducing cell death (31). In addition, silencing of VDAC1 expression in multiple types of cancer cell lines, including lung, prostate and colon cancer cells, has been demonstrated to block proliferation of the cancer cells and tumor growth in vivo and in vitro (20). The present data demonstrated that JQ1 decreased VDAC1 expression in a time- and dose- dependent manner in MCF-7 and T47D cells. These data indicate that JQ1 may directly regulate the expression of VDAC1 in breast cancer cells. It was further demonstrated that BRD4, a direct target of JQ1, could bind to the promoter region of VDACI and promote VDAC1 expression. Thus, the present findings appear to highlight a novel regulatory mechanism for VDAC1 in breast cancer.

A number of anticancer agents, including vorinostat and gefitinib, have been reported to induce the upregulation of VDAC1, resulting in the maintenance of mitochondrial 
function (32). This process may contribute to the observed resistance to anticancer drugs (33). Thus, VDAC1 may serve a key role in regulating chemotherapy drug resistance. Consistent with this, the current study demonstrated that knockdown of VDAC1 increased the sensitivity of breast cancer cells to JQ1. As acquired drug resistance to bromodomain inhibitors reduces the antitumor effect of these small molecule inhibitors, VDAC1 expression may be a valuable biomarker for evaluating the therapeutic effect of bromodomain inhibitors in the treatment of breast cancer. In the present study, JQ1 decreased VDAC1 expression, which was contradictory to the effect of VDAC1 on modulating the sensitivity of pancreatic cancer cells to JQ1. JQ1 blocks the function of bromodomain containing proteins, including BRD4, BRD2 and BRD3, decreases expression of the proto-oncogene Myc, and inhibits cancer cell growth and migration (19). However, the effects of JQ1 on breast cancer cells involve VDAC1-independent mechanisms, which have not been investigated in the current study.

In summary, the present study demonstrated that VDAC1 promotes the growth of tumor cells in breast cancer. These data suggest that JQ1 inhibits the expression of VDAC1, and that BRD4 is capable of upregulating the expression of VDAC1 in breast cancer cells. It was also demonstrated that VDAC1 may be critical for JQ1 resistance in breast cancer cells. These findings reveal important aspects of the function of VDAC1 in breast cancer cell progression, and elucidate the specific mechanism directing VDAC1 expression.

\section{Acknowledgements}

Not applicable.

\section{Funding}

No funding was received.

\section{Availability of data and materials}

The datasets generated/analyzed in the present study are available upon reasonable request from the corresponding author.

\section{Authors' contributions}

HC conceived and designed the study. GY and DZ performed experiments. JL and WW collected the data. WZ, WF and MY interpreted the data. HC analyzed the data and wrote the manuscript. All authors reviewed the results and approved the final version of the manuscript.

\section{Ethics approval and consent to participate}

Not applicable.

\section{Patient consent for publication}

Not applicable.

\section{Competing interests}

The authors declare that they have no competing interests.

\section{References}

1. Global Burden of Disease Cancer Collaboration; Fitzmaurice C, Allen C, Barber RM, Barregard L, Bhutta ZA, Brenner H, Dicker DJ, Chimed-Orchir O, Dandona R, et al: Global regional and national cancer incidence mortality years of life lost years lived with disability, and disability-adjusted life-years for 32 cancer groups, 1990 to 2015: A systematic analysis for the global burden of disease study. JAMA Oncol 3: 524-548, 2017.

2. Pathmanathan N and Balleine RL: Ki67 and proliferation in breast cancer. J Clin Pathol 66: 512-516, 2013.

3. Dong LF and Neuzil J: Mitochondria in cancer: Why mitochondria are a good target for cancer therapy. Prog Mol Biol Transl Sci 127: 211-227, 2014.

4. Shoshan-Barmatz V, Krelin Y, Shteinfer-Kuzmine A and Arif T: Voltage-dependent anion channel 1 as an emerging drug target for novel anti-cancer therapeutics. Front Oncol 7: 154, 2017.

5. Shoshan-Barmatz V, De Pinto V, Zweckstetter M, Raviv Z, Keinan N and Arbel N: VDAC, a multi-functional mitochondrial protein regulating cell life and death. Mol Aspects Med 31: 227-285, 2010.

6. Lemasters JJ: Evolution of voltage-dependent anion channel function: From molecular sieve to governator to actuator of ferroptosis. Front Oncol 7: 303, 2017.

7. Shoshan-Barmatz V, Nahon-Crystal E, Shteinfer-Kuzmine A and Gupta R: VDAC1, mitochondrial dysfunction, and alzheimer's disease. Pharmacol Res 131: 87-101, 2018.

8. Pittala S, Krelin Y and Shoshan-Barmatz V: Targeting liver cancer and associated pathologies in mice with a mitochondrial VDAC1-Based peptide. Neoplasia 20: 594-609, 2018.

9. Zhang G, Jiang G, Wang C, Zhong K, Zhang J, Xue Q, Li X, Jin H and Li B: Decreased expression of microRNA-320a promotes proliferation and invasion of non-small cell lung cancer cells by increasing VDAC1 expression. Oncotarget 7: 49470-49480, 2016.

10. Zhang C, Ding W, Liu Y, Hu Z, Zhu D, Wang X, Yu L, Wang L, Shen $\mathrm{H}$, Zhang W, et al: Proteomics-based identification of VDAC1 as a tumor promoter in cervical carcinoma. Oncotarget 7: 52317-52328, 2016

11. Magri A, Reina S and De Pinto V: VDAC1 as pharmacological target in cancer and neurodegeneration: Focus on its role in apoptosis. Front Chem 6: 108, 2018

12. Reina S and De Pinto V: Anti-cancer compounds targeted to VDAC: Potential and perspectives. Curr Med Chem 24: 4447-4469, 2017.

13. Sahni JM and Keri RA: Targeting bromodomain and extraterminal proteins in breast cancer. Pharmacol Res 129: 156-176, 2018.

14. Dai X, Gan W, Li X, Wang S, Zhang W, Huang L, Liu S, Zhong Q, Guo J, Zhang J, et al: Prostate cancer-associated SPOP mutations confer resistance to BET inhibitors through stabilization of BRD4. Nat Med 23: 1063-1071, 2017.

15. Decker TM, Kluge M, Krebs S, Shah N, Blum H, Friedel CC and Eick D: Transcriptome analysis of dominant-negative Brd4 mutants identifies Brd4-specific target genes of small molecule inhibitor JQ1. Sci Rep 7: 1684, 2017.

16. Jin X, Yan Y, Wang D, Ding D, Ma T, Ye Z, Jimenez R, Wang L, Wu $\mathrm{H}$ and Huang H: DUB3 Promotes BET Inhibitor resistance and cancer progression by deubiquitinating BRD4. Mol Cell 71: 592-605.e4, 2018.

17. Delmore JE, Issa GC, Lemieux ME, Rahl PB, Shi J, Jacobs HM, Kastritis E, Gilpatrick T, Paranal RM, Qi J, et al: BET bromodomain inhibition as a therapeutic strategy to target c-Myc. Cell 146: 904-917, 2011.

18. Livak KJ and Schmittgen TD: Analysis of relative gene expression data using real-time quantitative PCR and the 2(-Delta Delta C(T)) method. Methods 25: 402-408, 2001.

19. Zhang P, Wang D, Zhao Y, Ren S, Gao K, Ye Z, Wang S, Pan CW, Zhu Y, Yan Y, et al: Intrinsic BET inhibitor resistance in SPOP-mutated prostate cancer is mediated by BET protein stabilization and AKT-mTORC1 activation. Nat Med 23: 1055-1062, 2017.

20. Arif T, Vasilkovsky L, Refaely Y, Konson A and Shoshan-Barmatz V: Silencing VDAC1 Expression by siRNA Inhibits cancer cell proliferation and tumor growth in vivo. Mol Ther Nucleic Acids 3: e159, 2014.

21. Freitas S, Martins R, Costa M, Leão PN, Vitorino R, Vasconcelos V and Urbatzka R: Hierridin B isolated from a marine cyanobacterium alters VDAC1, mitochondrial activity, and cell cycle genes on HT-29 Colon adenocarcinoma cells. Mar Drugs 14: pii: E158, 2016. 
22. Maimaiti A, Aili A, Kuerban H and Li X: VDAC1 Mediated anticancer activity of gallic acid in human lung adenocarcinoma A549 cells. Anticancer Agents Med Chem 18: 255-262, 2018.

23. Thinnes FP: Neuroendocrine differentiation of LNCaP cells suggests: VDAC in the cell membrane is involved in the extrinsic apoptotic pathway. Mol Genet Metab 97: 241-243, 2009.

24. Tang Z, Li C, Kang B, Gao G, Li C and Zhang Z: GEPIA: A web server for cancer and normal gene expression profiling and interactive analyses. Nucleic Acids Res 45: W98-W102, 2017.

25. Shoshan-Barmatz V, Israelson A, Brdiczka D and Sheu SS: The voltage-dependent anion channel (VDAC): Function in intracellular signalling, cell life and cell death. Curr Pharm Des 12: 2249-2270, 2006.

26. Shoshan-Barmatz V and Mizrachi D: VDAC1: From structure to cancer therapy. Front Oncol 2: 164, 2012.

27. Seyfried TN and Shelton LM: Cancer as a metabolic disease. Nutr Metab (Lond) 7: 7, 2010.

28. Weisthal S, Keinan N,Ben-Hail D, ArifT and Shoshan-Barmatz V: $\mathrm{Ca}(2+)$-mediated regulation of VDAC1 expression levels is associated with cell death induction. Biochim Biophys Acta 1843: 2270-2281, 2014

29. Castagna A, Antonioli P, Astner H, Hamdan M, Righetti SC, Perego P, Zunino F and Righetti PG: A proteomic approach to cisplatin resistance in the cervix squamous cell carcinoma cell line A431. Proteomics 4: 3246-3267, 2004.
30. Jung JY, Han CR, Jeong YJ, Kim HJ, Lim HS, Lee KH, Park HO, Oh WM, Kim SH and Kim WJ: Epigallocatechin gallate inhibits nitric oxide-induced apoptosis in rat PC12 cells. Neurosci Lett 411: 222-227, 2007.

31. Nawarak J, Huang-Liu R, Kao SH, Liao HH, Sinchaikul S, Chen ST and Cheng SL: Proteomics analysis of A375 human malignant melanoma cells in response to arbutin treatment. Biochim Biophys Acta 1794: 159-167, 2009.

32. Leone A, Roca MS, Ciardiello C, Terranova-Barberio M, Vitagliano C, Ciliberto G, Mancini R, Di Gennaro E, Bruzzese F and Budillon A: Vorinostat synergizes with EGFR inhibitors in NSCLC cells by increasing ROS via up-regulation of the major mitochondrial porin VDAC1 and modulation of the c-Myc-NRF2-KEAP1 pathway. Free Radic Biol Med 89: 287-299, 2015.

33. Bhat TA, Kumar S, Chaudhary AK, Yadav N and Chandra D: Restoration of mitochondria function as a target for cancer therapy. Drug Discov Today 20: 635-643, 2015.

This work is licensed under a Creative Commons Attribution-NonCommercial-NoDerivatives 4.0 International (CC BY-NC-ND 4.0) License. 